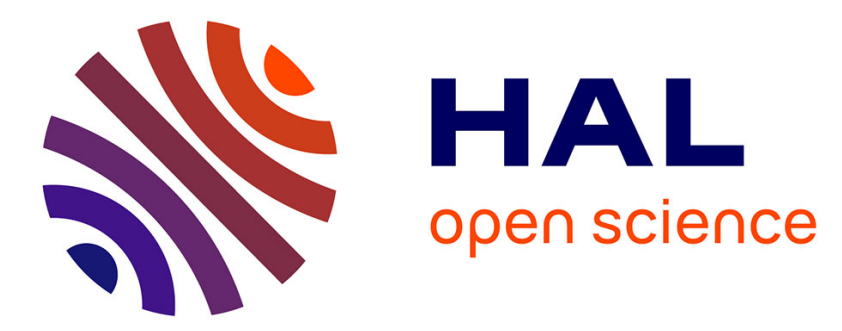

\title{
Dynamique des perceptions de momentum psychologique en situations d'accomplissement chez des acteurs virtuels
}

Walid Briki, Christophe Gernigon

\section{- To cite this version:}

Walid Briki, Christophe Gernigon. Dynamique des perceptions de momentum psychologique en situations d'accomplissement chez des acteurs virtuels. Annee Psychologique, 2015, 115 (02), pp.265-287. 10.4074/S0003503314000098 . hal-02520963

\section{HAL Id: hal-02520963 \\ https://hal.science/hal-02520963}

Submitted on 27 Mar 2020

HAL is a multi-disciplinary open access archive for the deposit and dissemination of scientific research documents, whether they are published or not. The documents may come from teaching and research institutions in France or abroad, or from public or private research centers.
L'archive ouverte pluridisciplinaire HAL, est destinée au dépôt et à la diffusion de documents scientifiques de niveau recherche, publiés ou non, émanant des établissements d'enseignement et de recherche français ou étrangers, des laboratoires publics ou privés. 
See discussions, stats, and author profiles for this publication at: https://www.researchgate.net/publication/279219535

Dynamique des perceptions de momentum psychologique en situations d'accomplissement chez des acteurs virtuels

Article in L?Année psychologique · June 2014

DOI: $10.4074 / 50003503314000098$

CITATION

1

2 authors:

Walid Briki

Qatar University

51 PUBLICATIONS 397 CITATIONS

SEE PROFILE

Some of the authors of this publication are also working on these related projects:

Counterfactual Thinking and Imagination Page View project

Project Understanding Psychological Momentum View project
READS

74

Christophe Gernigon

Université de Montpellier

70 PUBLICATIONS 871 CITATIONS

SEE PROFILE 


\title{
Dynamique des perceptions de momentum psychologique en situations d'accomplissement chez des acteurs virtuels
}

\author{
Walid Briki $^{{ }^{*}}$ et Christophe Gernigon ${ }^{2}$ \\ ${ }^{1}$ Université des Antilles et de la Guyane, Département des Sciences du Sport, Laboratoire \\ ACTES, Pointe-à-Pitre, France \\ ${ }^{2}$ Université Montpellier I, Département des Sciences du Sport et de l'Éducation Physique, \\ Laboratoire Epsylon, Montpellier, France
}

\begin{abstract}
RÉSUMÉ
Empruntant l'approche des systèmes dynamiques, la présente recherche avait pour but d'examiner les changements dans les perceptions de momentum psychologique (MP) en situations d'accomplissement virtuelles. Les participants devaient visionner une manche de tennis de table et se projeter à la place d'un joueur en train de vivre une séquence de score ascendante (c'est-à-dire, momentum positif) ou descendante (c'est-à-dire, momentum négatif). Les séquences de momentum utilisées contenaient des événements identiques présentés dans un ordre inverse d'une séquence de momentum à l'autre. À l'issue de chaque échange, les participants répondaient à des items de perceptions de MP présentés de manière aléatoire. Les perceptions de MP ont montré une variation abrupte en début des deux séquences de momentum suivie d'une stabilisation, donnant la forme d'un patron dynamique d'hystérésis négative tel celui déjà observé par Gernigon et al. (2010). La présente recherche incite à poursuivre l'examen de la dynamique du MP.
\end{abstract}

Dynamics of perceptions of psychological momentum

in achievement situations in virtual actors

ABSTRACT

Borrowing the dynamical systems approach, the present research aimed to examine changes in perceptions of psychological momentum (PM) during virtual achievement situations. The participants had to watch the video of table tennis game while empathizing

*Correspondance : Walid Briki, Université des Antilles et de la Guyane, Département des Sciences du Sport, Laboratoire ACTES, Guadeloupe, Pointe-à-Pitre, Campus Fouillole, 97159, France. E-mail : walid.briki@univag.fr, briki.w@gmail.com 
with a player who was experiencing either an ascending (i.e., positive momentum) or descending (i.e., negative momentum) scoring sequence. The momentum sequences that were used included same events that were presented in a reversed order according to the momentum sequence. Following each exchange, participants answered PM perceptions items that were presented randomly. PM perceptions displayed an abrupt change followed by stabilization, displaying a pattern of negative hysteresis as already observed by Gernigon et al. (2010). The present work incites to pursue the examination of the dynamics of PM.

\section{INTRODUCTION}

Le Momentum psychologique (MP) désigne cette force psychologique qui modifie la manière dont les événements sont vécus (par ex., Adler, 1981 ; Jones \& Harwood, 2008). Le MP peut être positif ou négatif et renvoie respectivement à une sorte d'élan traduisant le fait de bénéficier d'une spirale de succès ou d'être aspiré dans une spirale d'échec. Le scénario fictif ci-dessous relate une expérience de MP négatif :

Dans cette finale, j'avais déjà gagné les deux premières manches et je menais 7-2 dans la troisième. Tout allait donc pour le mieux dans le meilleur des mondes. Mais il y a eu ce point interminable, âprement disputé, où toutes mes attaques étaient retournées avec succès, parfois avec un peu de chance pour lui. Et puis, au bout d'un nombre invraisemblable de coups pour rien, ce smash que j'ai balancé dans le filet, sans trop savoir si c'était vraiment pour gagner le point et asseoir ma domination sur l'autre ou si c'était pour mettre fin à cet épuisement et cette tension qui me gagnaient alors que l'échange traînait en longueur. En revanche, après ce point, dans ma tête, rien n'était plus comme avant. Un ressort avait cédé ! J'étais fébrile, mon poignet était tendu, mes coups étaient trop forts, c'était un autre match!

Initialement, le MP a été conceptualisé comme un phénomène dynamique profondément enraciné dans les interactions sociales (Adler, 1981). Cherchant à explorer la dynamique du MP, des auteurs ont révélé que le MP pouvait montrer des variations abruptes ou graduelles au cours du temps (Briki, Den Hartigh, Bakker, \& Gernigon, 2012) et était inscrit 
dans une histoire en cours des événements spécifiques (Briki, Den Hartigh, Hauw, \& Gernigon, 2012). En cohérence avec de telles conceptions, Gernigon, Briki et Eykens (2010) ont conceptualisé le MP en empruntant l'approche des systèmes dynamiques. Dans cette perspective, ces auteurs ont défini le MP comme une «[...] dynamique positive ou négative des réponses cognitives, affectives, motivationnelles, physiologiques et comportementales (et leurs couplages) à la perception de progression ou d'éloignement vis-à-vis d'une issue appétitive ou aversive » (p. 397). Le $\mathrm{MP}$ reflète un phénomène global touchant aux sphères affective, cognitive, physiologique et comportementale. Le sentiment de faire l'expérience du $\mathrm{MP}$ renvoie à la notion de MP perçu.

\subsection{L'Approche des Systèmes Dynamiques Appliquée au MP}

Un « système dynamique » correspond à un ensemble d'éléments interconnectés soumis au changement au cours du temps (par ex., Nowak \& Vallacher, 1998). Un tel système est reconnaissable à certaines de ses propriétés dynamiques (par ex., Kelso, 1995 ; Nowak \& Vallacher, 1998). Parmi ces propriétés, la «non-linéarité » et la « dépendance à l'histoire » ont été récemment identifiées comme des propriétés dynamiques du MP (Briki, Den Hartigh, Markman, Micallef, \& Gernigon, 2013 ; Briki, Den Hartigh, Markman, \& Gernigon, 2014 ; Gernigon et al., 2010). La «non-linéarité » renvoie à la tendance du système à montrer des changements qualitatifs abrupts dans son état global au cours du temps. Mathématiquement, un système est non-linéaire lorsque les valeurs à son entrée (input) et sa sortie (output) n'entretiennent pas de rapports proportionnels systématiques. Ainsi, une grande variation dans la valeur d'un ou plusieurs éléments du système peut n'entraîner aucun changement dans le phénomène en question (lorsque l'état du système est stable), alors qu'une infime variation dans la valeur de l'un des éléments du système peut entraîner un changement catastrophique de son comportement global (lorsque l'état du système est instable). La " dépendance à l'histoire », quant à elle, signifie que l'état d'un système à un moment donné dépend de sa propre histoire (c'est-à-dire, de ses états précédents), entraînant ainsi une résistance au changement. Une telle résistance refléterait l'existence d'un «attracteur », désignant « [...] un état ou un patron de changement fiable vers lequel un système dynamique évolue au cours du temps et vers lequel le système retourne après avoir été perturbé » (Nowak, Vallacher, \& Zochowski, 2005, p. 354). 
Afin de tester les propriétés de non-linéarité et de dépendance à l'histoire des systèmes dynamiques, certains théoriciens des systèmes dynamiques (Haken, Kelso, \& Bunz, 1985) ont recommandé de tester l'effet des variations graduelles - dans le sens ascendant et descendant - d'un paramètre de contrôle sur le paramètre d'ordre d'un système dynamique. Un paramètre de contrôle est une variable capable de conduire le système à ses différents états possibles, sans prescrire l'émergence de ces états (par ex., Kelso, 1995). Un paramètre d'ordre correspond à une variable capable de refléter, de rendre compte de l'état global du système (par ex., Kelso, 1995 ; Nowak \& Vallacher, 1998). Appliquant les recommandations de Haken et al. (1985), des auteurs ont identifié trois catégories de patron dynamique (par ex., voir Briki, Den Hartigh, Markman, \& Gernigon, 2014 ; Tuller, Case, Ding, \& Kelso, 1994). Un patron d'hystérésis positive est observé lorsque le système résiste aux perturbations de telle sorte que les changements du paramètre d'ordre, résultant des variations ascendantes et descendantes du paramètre de contrôle, sont retardés (voir Figure 1A). Un patron d'hystérésis négative est observé lorsque le système varie en réponse aux perturbations de telle sorte que le paramètre d'ordre varie au début des deux séquences expérimentales (voir Figure 1B). Un patron de limite critique est observé lorsque le système varie en réponse à une valeur spécifique du paramètre de contrôle de telle sorte que le paramètre d'ordre change en réponse à la même valeur du paramètre de contrôle au cours des deux séquences expérimentales (voir Figure 1C).

Basés sur les recommandations de Haken et al. (1985), Gernigon et al. (2010) et Briki, Den Hartigh, Markman et al. (2013) ont tenté d'investiguer les propriétés dynamiques du MP. Le MP était considéré comme un système dynamique, caractérisé par la présence de deux attracteurs - le MP positif et le MP négatif - pouvant émerger des interactions complexes entre les multiples déterminants du MP. Gernigon et al. (2010) ont investigué l'influence de séquences de score ascendante (c'est-à-dire, momentum positif) et descendante (c'est-à-dire, momentum négatif) sur les fluctuations de l'anxiété compétitive, de la confiance en soi (Étude 1) et des buts d'accomplissement (Étude 2) pendant que les participants se projetaient à la place de l'un des pongistes (visibles dans une vidéo) disputant une manche de haute importance. Ces variables étaient considérées comme des paramètres d'ordre du MP. À l'issue de chaque échange, les participants répondaient à des questions relatives aux variables examinées sur une échelle de type Lickert.

L'anxiété compétitive a révélé un patron de MP positif caractérisé par une évolution graduelle en séquence de momentum positif et un 


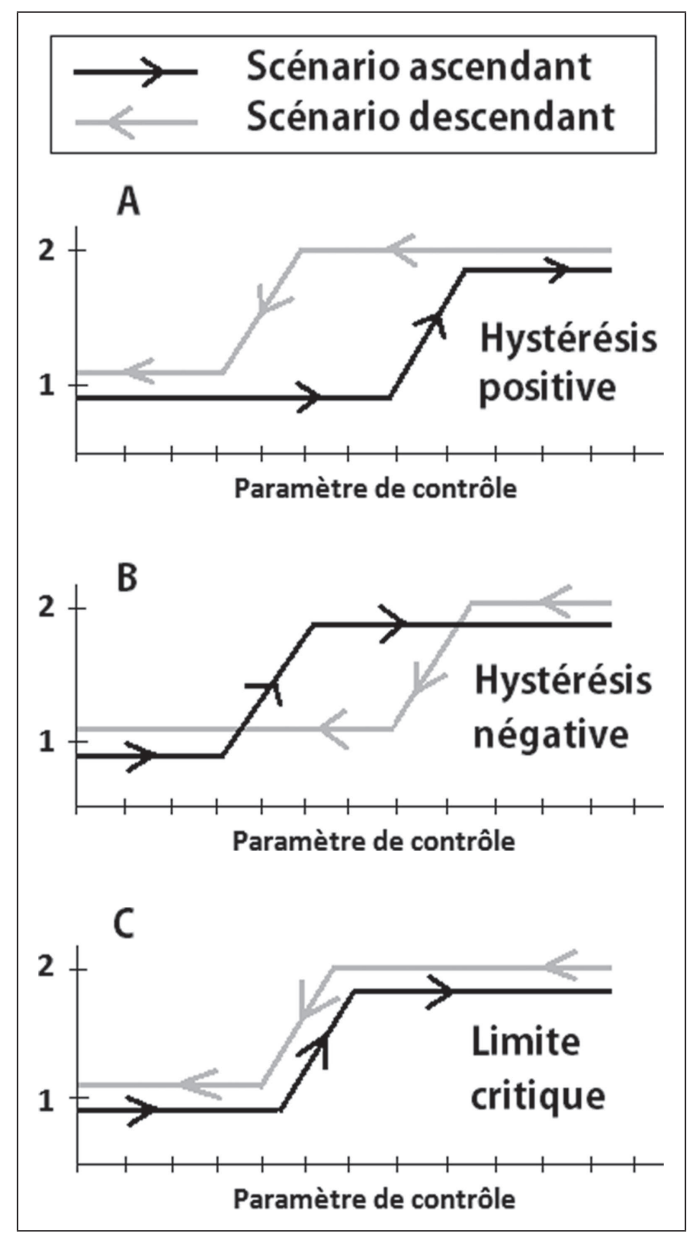

Figure 1. Représentation schématique des patrons dynamiques (A) d'hystérésis positive, (B) d'hystérésis négative et (C) de limite critique. Le scénario ascendant correspond à un scénario dans lequel le paramètre de contrôle varie de manière ascendante (c'est-à-dire, des valeurs basses à des valeurs élevées), alors que le scénario descendant correspond à un scénario dans lequel le paramètre de contrôle varie de manière descendante (c'est-à-dire, des valeurs élevées à des valeurs basses). Sur l'axe y, les chiffres 1 et 2 représentent des états possibles d'un système composé de deux attracteurs.

Figure 1. Schematic representation of the dynamical patterns of (A) positive hysteresis, (B) negative hysteresis, and (C) critical boundary. The ascending scenario corresponds to a scenario in which the control parameter changes in an ascending way (i.e., from low values to high values), whereas the descending scenario corresponds to a scenario in which the control parameter varies in a descending way (i.e., from high values to low values). On the y-axis, the numbers 1 and 2 represent possible states of a system composed of two attractors. 
patron de MP négatif caractérisé par une évolution abrupte en séquence de momentum négatif. Cette asymétrie soutient l'hypothèse que le MP négatif serait plus facile à déclencher que le MP positif (Adler, 1981). Plus généralement, ce résultat est cohérent avec l'asymétrie entre les gains et les pertes décrite par Kahneman et Tversky (1979) dans leur théorie de la perspective. D'après ces auteurs, la fonction de valeur est plus pentue pour les pertes que pour les gains. Cette asymétrie est conforme au fait persistant que les événements négatifs ont plus d'impact au plan psychologique que des événements positifs équivalents (Baumeister, Bratslavsky, Finkenauer, \& Vohs, 2001). Concernant les buts d'accomplissement, les buts de maîtrise-évitement (c'est-à-dire, consistant à éviter de faire des erreurs) ont montré des patrons de MP caractérisés par des changements abrupts dans les séquences de momentum positif et négatif, dans la forme d'un patron d'hystérésis négative. Gernigon et al. (2010) ont suggéré que ce patron dynamique refléterait la propension des individus à se projeter dans un futur probable de la situation dans laquelle ils sont impliqués, ceci sur la base du moindre événement contradictoire avec l'état actuel de la situation.

Briki, Den Hartigh, Markman et al. (2013) ont examiné la dynamique des perceptions du MP en contexte de compétitions de cyclisme sur home-trainers. Deux avatars en mouvement des cyclistes étaient projetés sur un écran placé en face des deux coureurs dans le but de leur fournir des feed-backs de performance (manipulés). Les feed-backs étaient manipulés de sorte que l'un des coureurs était exposé à une séquence de momentum positif (c'est-à-dire, évoluant du retard à la prise d'avance), pendant que l'autre coureur était exposé à une séquence de momentum négatif (c'est-à-dire, évoluant de l'avance à la prise de retard). Les perceptions de MP des cyclistes étaient alors mesurées périodiquement à l'aide d'un clavier numérique disposé sur le guidon de chaque bicyclette (par ex., Qui te semble avoir le plus le vent en poupe ? [1] «lui », [2] « moi »). Les perceptions de MP ont révélé un patron de limite critique. Cependant, ce patron de limite critique était asymétrique dans le sens où le changement observé correspondait à une diminution anticipée des perceptions de MP durant la séquence de momentum négatif et à une augmentation retardée durant la séquence de momentum positif. Parce que le MP négatif a persisté plus longtemps que le MP positif, Briki, Den Hartigh, Markman et al. (2013) ont suggéré que le MP négatif pouvait correspondre à un attracteur plus fort que le MP positif.

Les résultats des études précédentes ont apporté un support aux hypothèses selon lesquelles (a) le MP possède des propriétés de non-linéarité et de dépendance à l'histoire (Briki, Den Hartigh, Bakker, \& Gernigon, 
2012 ; Briki, Den Hartigh, Hauw, \& Gernigon, 2012), et (b) le MP négatif est déclenché plus facilement que le MP positif (Adler, 1981). Cependant, des incohérences dans les résultats des différentes études peuvent être observées. Premièrement, le travail de Gernigon et al. (2010) a produit des résultats disparates montrant tantôt le caractère dissymétrique de l'anxiété compétitive, tantôt un patron dynamique d'hystérésis négative pour les buts de maîtrise-évitement. Deuxièmement, le patron de limite critique observé par Briki, Den Hartigh, Markman et al. (2013) est incohérent avec le patron d'hystérésis négative observé par Gernigon et al. (2010). Comme le supposent Briki, Den Hartigh, Markman et al. (2013), ces incohérences de résultats pourraient être imputées à des différences méthodologiques. Parmi ces différences, Briki, Den Hartigh, Markman et al. (2013) ont pointé l'effet différencié potentiel du type d'implication dans la tâche expérimentale : alors que Gernigon et al. (2010) ont placé des participants en position d'acteur projeté dans des situations virtuelles, Briki, Den Hartigh, Markman et al. (2013) ont placé des participants en position d'acteur en situations réelles de production de performance. Briki, Den Hartigh, Markman et al. (2013) ont aussi pointé l'utilisation de variables psychologiques différentes : alors que Gernigon et al. (2010) ont utilisé des variables réputées pour être des contenus du MP (voir Briki, Den Hartigh, Hauw, \& Gernigon, 2012), Briki, Den Hartigh, Markman et al. (2013) ont mesuré les perceptions de MP réputées pour être les mieux à même de capturer le MP dans sa globalité (par ex., voir Markman \& Guenther, 2007 ; Vallerand, Colavecchio, \& Pelletier, 1988).

\subsection{La Présente Recherche}

Le présent travail avait pour but de faire la lumière sur les incohérences de résultats mentionnées plus haut. Plus spécifiquement, le premier but de ce travail était d'examiner la dynamique du MP afin de confirmer ses propriétés de non-linéarité et de dépendance à l'histoire, en exhibant notamment des phénomènes d'anticipation (Briki, Den Hartigh, Markman et al., 2013; Gernigon et al., 2010) et/ou de persistance (Briki, Den Hartigh, Markman et al., 2013) durant les séquences de momentum. Le deuxième but de ce travail était de tester l'hypothèse selon laquelle le MP négatif est déclenché plus facilement que le MP positif (Briki, Den Hartigh, Markman et al., 2013 ; Gernigon et al., 2010). Le MP était examiné au travers des perceptions de MP parce que cette variable a été considérée comme un paramètre d'ordre fiable du MP (Briki, Den Hartigh, Markman et al., 2013). 
Deux études expérimentales ${ }^{1}$, basées sur les séquences de tennis de table utilisées par Gernigon et al. (2010), ont composé le présent travail. Parce que le MP se développerait d'autant plus qu'un individu accorderait de l'importance à la situation (Markman \& Guenther, 2007), les séquences de score de tennis de table utilisées étaient situées dans un contexte à fort enjeu.

\section{2. ÉTUDE 1}

\subsection{Méthode}

\subsubsection{Participants}

Cinquante-et-un étudiants hommes en faculté des sciences du sport de l'université Montpellier I ont été volontaires pour participer à cette étude en l'échange d'aucune rémunération. Le recrutement de ces participants s'était réalisé sur la base de leur disponibilité. Tous étaient des sportifs compétiteurs dans différentes activités sportives autres que celle du tennis de table. Ces participants avaient une moyenne d'âge de 22,2 ans $(s=1,22)$.

\subsubsection{Dispositif expérimental}

Le dispositif expérimental était installé dans une salle de l'université de Montpellier I. Ce dispositif comportait un ordinateur portable et un vidéoprojecteur placés sur une table derrière laquelle les participants étaient assis. La table était située à $2,5 \mathrm{~m}$ du mur sur lequel les images (de dimension $1,5 \mathrm{~m}$ de largeur $\times 1,5 \mathrm{~m}$ de hauteur) étaient projetées. Un stylo et une pile de fiches (au format A5) sur lesquelles figuraient les items étaient aussi placés sur la table. L'ordinateur portable comportait les scénarios de tennis de table visionnés (voir Figure 2A). Ces scénarios étaient issus d'un montage vidéo d'un match filmé lors d'une compétition de niveau régional qui s'était déroulée en région parisienne, opposant des joueurs inconnus ${ }^{2}$ des participants de l'étude. Ce match a été enregistré simultanément par deux caméras placées de telle sorte qu'elles offraient une perspective de trois-quart-dos de l'un ou l'autre des deux joueurs. Ainsi, chaque point pouvait être visionné dans la perspective de l'un ou de l'autre joueur (voir Figures 2B et 2C). Ensuite, certains points du match ont été sélectionnés et leurs séquences vidéo ont été montées pour construire les séquences de score ascendante (c'est-à-dire, séquence

${ }^{1}$ La présente recherche a été réalisée dans le respect de la déclaration d'Helsinki dans la mesure où elle ne portait aucune atteinte à la vie, la santé, la dignité et l'intimité des participants. Les données des deux études ayant fait l'objet d'analyses statistiques peuvent être réclamées aux auteurs.

${ }^{2}$ Étant donné que le match de tennis de table de niveau régional s'était déroulé en région parisienne, nous avions supposé que les pongistes visibles à l'écran étaient inconnus des participants qui résidaient dans le sud de la France et qui n'avaient jamais pratiqué le tennis de table en compétitions. Les participants ont tous confirmé cette supposition. 


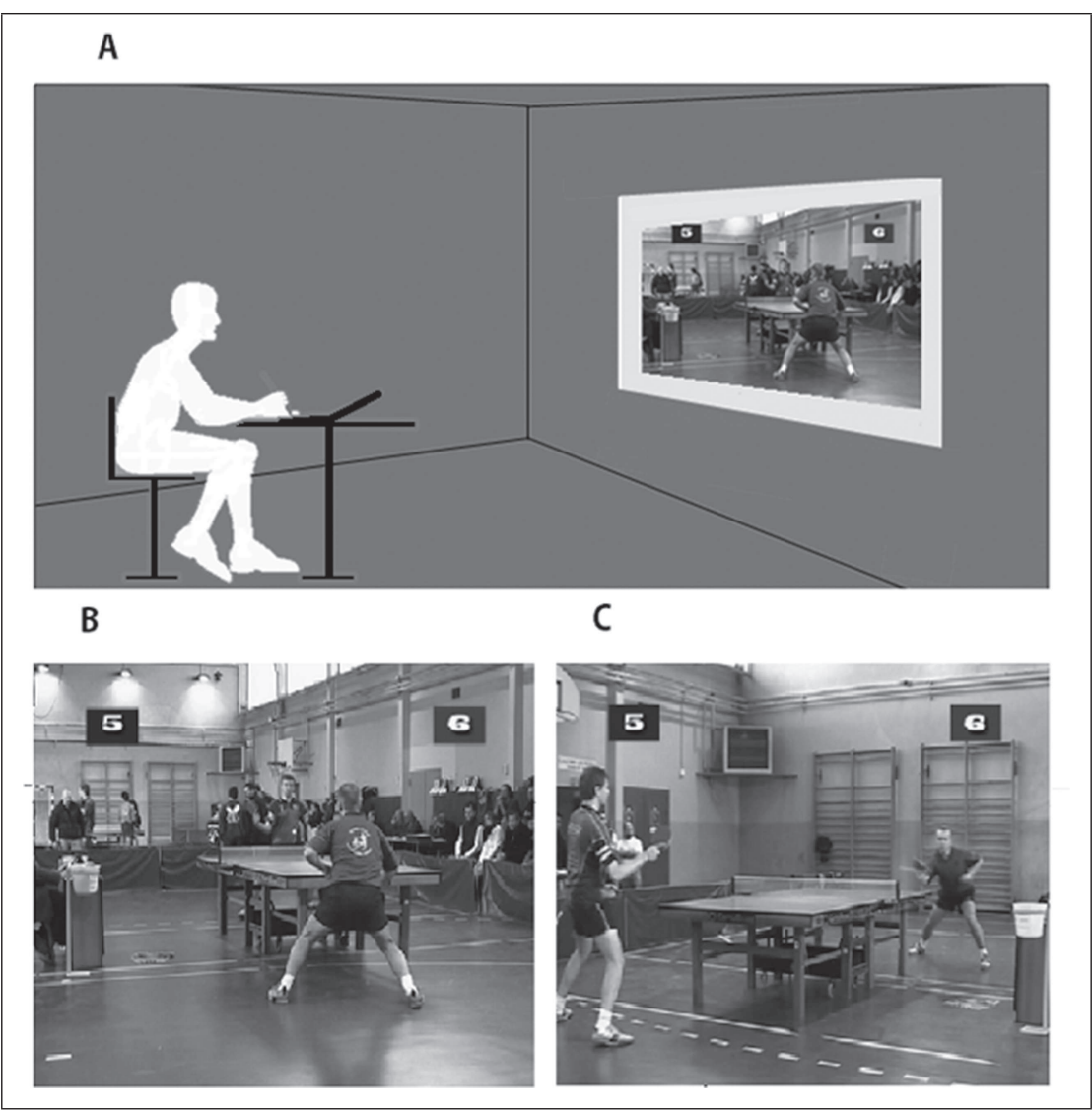

Figure 2. (A) Représentation schématique de l'installation du dispositif expérimental, et exemples de prise de vue de la caméra selon les perspectives des séquences (B) de momentum positif et $(\mathrm{C})$ de momentum négatif. Cet instant du montage vidéo présente un échange entre les deux joueurs lorsque le score était de 6 à 5 en faveur du futur vainqueur, correspondant à l'écart de score +1 (ou à l'écart de score -1) en séquence de momentum positif (ou en séquence de momentum négatif).

Figure 2. (A) Schematic representation of installation of the experimental design, and examples of camera's angles of view according to (B) positive and (C) negative momentum sequences. This moment of the video-tape presents an exchange between the both players when the score was 6-5 in favor of the future winner, corresponding to the score gap +1 (or to the score gap -1) in the positive momentum sequence (or in the negative momentum sequence). 
de momentum positif) et descendante (c'est-à-dire, séquence de momentum négatif). En conséquence, les deux séquences de momentum concernaient les mêmes échanges et étaient parfaitement symétriques en termes de contenu. La seule différence était que dans le scénario comportant la séquence de momentum positif, le joueur visible de dos, au premier plan, était le futur vainqueur, alors que dans le scénario comportant la séquence de momentum négatif le joueur situé au premier plan était le futur perdant (voir Figures $2 \mathrm{~B}$ et $2 \mathrm{C}$ ).

Les deux scénarios débutaient au score de $0-0$ et se terminaient au score de 11-5 (ou 5-11). Après chaque point, apparaissaient sur tout l'écran deux panneaux rappelant les scores des deux joueurs. Chaque panneau était disposé sur la moitié gauche ou droite de l'écran en fonction du côté que le joueur correspondant occupait. Les deux scénarios incluaient une période d'amorçage au cours de laquelle le futur vainqueur (ou perdant) commençait par prendre un retard (ou une avance) de cinq points. Ainsi, la période concernée par l'étude (c'est-à-dire, la séquence de momentum) a consisté pour ce joueur à évoluer graduellement d'un écart de score négatif (ou positif), soit -5 (ou +5$)$, à un écart de score positif (ou négatif), soit +5 (ou -5), pour terminer le match par la victoire (ou la défaite). Les évolutions des écarts de score en fonction des séquences de momentum positif et négatif sont présentées dans le Tableau 1.

Tableau 1. Manipulation des écarts de score en fonction des scénarios de tennis de table

Table 1. Manipulation of score gaps according to table tennis scenarios

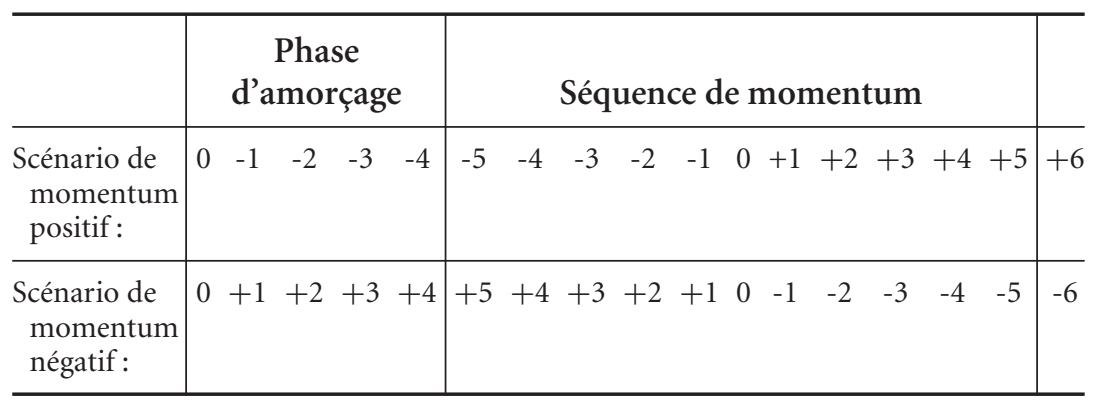

\subsubsection{Procédure}

Les participants ont été sollicités au sein de leur université, après avoir rempli et signé une fiche de consentement. L'expérimentation comportait une session correspondant à une condition de momentum positif $(n=28)$ ou à une condition de momentum négatif $(n=23)$. Les participants ont été assignés de manière aléatoire à l'une des deux conditions de séquence de momentum (positif vs négatif). 
Au cours de la session expérimentale, les participants étaient placés dans une salle sombre de l'université, dans laquelle le dispositif de l'expérimentation avait été préalablement installé.

Il était demandé à chaque participant (a) de visionner une manche d'un match de tennis de table, (b) de s'identifier au pongiste visible de dos, au premier plan, (c) de se projeter dans la manche décisive de la finale de la compétition la plus importante de sa carrière, et (d) d'imaginer que son adversaire et lui avaient tous deux gagné deux manches dans cette finale et qu'ils s'apprêtaient donc à entamer la manche décisive (appelée « la belle »). Puis, une fois que ces instructions étaient fournies, le participant commençait l'exécution de la tâche. Cette tâche consistait (a) avant chaque nouveau point de jeu, à prendre une fiche de la pile et, après y avoir indiqué le score en cours, à répondre aux items, (b) à visionner l'échange complet à la fin duquel apparaissaient deux panneaux indiquant le nouveau score des deux joueurs, (c) à reporter le score qui était inscrit à l'écran et à répondre aux items sur une nouvelle fiche, et ainsi de suite jusqu'à la fin du match. Afin de vérifier la bonne compréhension et le respect des instructions des participants, mais aussi dans le but de garantir le bon déroulement de l'expérimentation, un chercheur était resté dans la pièce pour la totalité de la manche.

\subsubsection{Mesures}

Parce qu'une interruption prolongée des séquences de momentum pourrait réduire leur impact sur les perceptions de MP des participants (voir Briki, Doron, Markman, Den Hartigh, \& Gernigon, 2014), un nombre limité d'items de perceptions de MP a été utilisé (par ex., Briki, Den Hartigh, Markman et al., 2013 ; Gernigon et al., 2010). Ces items, inspirés des items du questionnaire de perceptions de MP de Vallerand et al. (1988), ont été traduits selon la méthode de traduction inversée (Brislin, Lonner, \& Thorndike, 1973) et ont été adaptés pour correspondre aux expressions françaises idiomatiques typiques qui reflètent le MP : «Qui te semble avoir le plus le vent en poupe ? "; "Qui te semble progresser le plus vers la victoire ?»; «Qui te semble avoir le plus de contrôle sur la situation ? »; et «Qui te semble avoir le plus de chances de gagner ?» $(\alpha=0,89$; IC $95 \%[0,84 ; 0,93])$. Ces items ont été choisis en raison de leur compatibilité avec le contexte du tennis de table. Les questions étaient présentées de manière aléatoire et les participants y répondaient en cochant une des deux cases de la fiche correspondant aux réponses « lui » et « moi ». L'utilisation d'une modalité binaire dans le choix de réponse est conforme à la procédure utilisée par Briki et ses collaborateurs (Briki, Den Hartigh, Markman, \& Gernigon, 2014 ; Briki, Den Hartigh, Markman et al., 2013). Ce choix méthodologique avait pour but d'observer le plus clairement possible le changement dans l'état global du MP perçu. On retrouve cette binarité dans le choix de réponse dans certains travaux de psychologie cognitive adoptant l'approche des systèmes dynamiques, notamment 
dans le domaine de la catégorisation perceptive (par ex., Kelso, 1995 ; Tuller, Case, Ding, \& Kelso, 1994). Conformément aux prescriptions de Tabachnick et Fidell (2007) à l'égard des données dichotomiques, les réponses « lui » et « moi » ont été codées par le chiffre 1 et le chiffre 2, respectivement. Pour chaque écart de score, l'état de perception de MP correspondait à la moyenne des scores aux quatre items.

\subsubsection{Analyses}

Afin d'examiner la dynamique des perceptions de MP, des ANOVAs $2 \times 11$ (Séquence de momentum : positif vs négatif $\times$ Écart de score [ES] : $\mathrm{ES}_{-5}, \mathrm{ES}_{-4}$, $\mathrm{ES}_{-3}, \mathrm{ES}_{-2}, \mathrm{ES}_{-1}, \mathrm{ES}_{0}, \mathrm{ES}_{+1}, \mathrm{ES}_{+2}, \mathrm{ES}_{+3}, \mathrm{ES}_{+4}$ et $\mathrm{ES}_{+5}$ ) à mesures répétées sur l'Écart de score ont été réalisées sur les perceptions de MP qui ont été enregistrées au cours des séquences de momentum. Les ANOVAs ont été précédées par un test de sphéricité de Mauchly visant à vérifier l'homogénéité des variances. Lorsque le test était significatif $(p<0,05)$ - indiquant une violation de l'hypothèse de l'homogénéité des variances - une procédure de correction de Greenhouse-Geisser était utilisée afin d'ajuster les degrés de liberté.

\subsubsection{Caractéristiques du changement}

Des comparaisons post hoc (tests de Scheffé) ont été conduites lorsque cela était nécessaire en vue d'identifier les changements significatifs. Des différences significatives entre les écarts de score distants (c'est-à-dire, non adjacents, séparés par au moins un écart de score) refléteraient des changements graduels dans les perceptions de MP, alors que des différences significatives apparaissant entre des écarts de score adjacents refléteraient des changements abrupts (voir Briki, Den Hartigh, Markman, \& Gernigon, 2014 ; Briki, Den Hartigh, Markman et al., 2013 ; Gernigon et al., 2010). Cependant, les changements graduels versus abrupts sont indépendants des changements linéaires versus non-linéaires. La linéarité (ou non-linéarité) fait référence à une relation proportionnelle (ou disproportionnelle) entre des causes et des conséquences. Plus concrètement, des changements similaires consécutifs (graduels ou abrupts) entre des écarts de score refléteraient une dynamique linéaire, étant donnée l'existence de changements continus et réguliers. En revanche, des changements graduels ou abrupts suivis ou précédés de stabilité refléteraient une dynamique non-linéaire (voir Briki, Den Hartigh, Markman, \& Gernigon, 2014 ; Briki, Den Hartigh, Markman et al., 2013 ; Gernigon et al., 2010).

\subsubsection{Caractéristiques des patrons dynamiques}

Pour supporter l'existence d'un patron d'hystérésis positive (ou négative), les variations non-linéaires devraient apparaître à des valeurs différentes de l'écart de score, selon la séquence de momentum positif versus négatif. Mathématiquement, l'hystérésis positive (ou négative) suppose qu'une valeur donnée de $x$ fournit deux valeurs de $y$, sauf quand $x$ est minimal ou maximal (voir Figures $1 \mathrm{~A}$ et 1B). Ainsi, les effets de la séquence de momentum devraient être significatifs 
seulement pour quelques écarts de score donnés localisés autour du milieu de cette séquence, et non à son commencement ni à sa fin. Concernant l'hystérésis positive, les perceptions de MP devraient montrer une variation abrupte vers la fin (ou à proximité de la fin) des deux séquences de momentum (c'est-à-dire, apparition d'un retard du paramètre d'ordre sur le paramètre de contrôle). Concernant l'hystérésis négative, les perceptions de MP devraient montrer une variation abrupte dès le début (ou à proximité du début) des deux séquences de momentum. Par ailleurs, pour supporter l'existence de limite critique, les variations non-linéaires devraient apparaître à des valeurs identiques de l'écart de score, selon la séquence de momentum positif versus négatif. Mathématiquement, la limite critique suppose qu'une valeur donnée de $x$ fournit toujours une valeur de $y$ (voir Figure 1C). Ainsi, les perceptions de MP devraient montrer une variation abrupte à une valeur spécifique du paramètre de contrôle au cours des deux séquences de momentum.

\subsubsection{Mise en évidence des patrons dynamiques}

Statistiquement, la mise en évidence du patron d'hystérésis positive requerrait l'existence (a) d'un effet d'interaction Séquence de momentum $\times$ Écart de score significatif, (b) de différences significatives entre les séquences de momentum vers le milieu de ces séquences seulement, mais pas à leur début ni à leur fin, (c) d'un effet principal significatif de la Séquence de momentum montrant que les perceptions de MP sont plus élevées en momentum négatif qu'en momentum positif, et (d) d'un effet principal significatif de l'Écart de score montrant des variations abruptes dans les perceptions de MP en fin (ou proche de la fin) de chaque séquence de momentum (voir Tableau 2). Comme pour l'hystérésis positive, la mise en évidence du patron d'hystérésis négative nécessiterait l'existence des effets principaux et d'interaction significatifs décrits ci-dessus. Cependant, le patron d'hystérésis négative révélerait (a) des perceptions de MP plus élevées en momentum positif qu'en momentum négatif et (b) des variations abruptes dans ces perceptions en début (ou proche du début) de chaque séquence de momentum (voir Tableau 2). Concernant la limite critique, le patron de résultat concerne (a) l'existence d'un effet d'interaction Séquence de momentum $\times$ Écart de score non significatif et (b) d'un effet principal significatif de l'Écart de score montrant des variations abruptes dans ces perceptions à une valeur identique aux deux séquences de momentum (voir Tableau 2).

Enfin, pour supporter l'asymétrie entre le MP positif et le MP négatif, les perceptions de MP devraient démarrer plus tôt en séquence de momentum négatif qu'en séquence de momentum positif. 
Tableau 2. Récapitulatif des critères statistiques associés aux patrons dynamiques

Table 2. Summary of statistic criteria related to the dynamical patterns

\begin{tabular}{ll}
$\begin{array}{l}\text { Patron } \\
\text { dynamique }\end{array}$ & \multicolumn{1}{c}{ Patron de résultats } \\
\hline Hystérésis positive & $\begin{array}{c}\text { Effet d'interaction Séquence de momentum } \times \text { Écart de score } \\
\text { significatif : mise en évidence de différences significatives } \\
\text { entre les écarts de score des séquences de momentum, au } \\
\text { milieu de ces séquences seulement. } \\
\text { Effet principal de la Séquence de momentum significatif : mise } \\
\text { en évidence d'un score moyen de perceptions de MP plus } \\
\text { élevé en momentum négatif qu'en momentum positif. } \\
\text { Effet principal de l'Écart de score : mise en évidence de } \\
\text { variations abruptes dans les perceptions de MP en fin (ou à } \\
\text { proximité de la fin) de chaque séquence de momentum. } \\
\text { Effet d'interaction Séquence de momentum } \times \text { Écart de score } \\
\text { significatif : mise en évidence de différences significatives } \\
\text { entre les écarts de score des séquences de momentum, au } \\
\text { milieu de ces séquences seulement. } \\
\text { Effet principal de la Séquence de momentum significatif : mise } \\
\text { en évidence d'un score moyen de perceptions de MP plus } \\
\text { élevé en momentum positif qu'en momentum négatif. } \\
\text { Effet principal de l'Écart de score : mise en évidence de } \\
\text { variations abruptes dans les perceptions de MP en début } \\
\text { (ou à proximité du début) de chaque séquence de } \\
\text { momentum. } \\
\text { Effet d'interaction Séquence de momentum } \times \text { Écart de score } \\
\text { non significatif. } \\
\text { Effet principal de l'Écart de score : mise en évidence de } \\
\text { variations abruptes dans les perceptions de MP à une valeur } \\
\text { identique des écarts de score aux deux séquences de } \\
\text { momentum. }\end{array}$ \\
Limite critique & \\
&
\end{tabular}

Note. La mise en évidence des patrons de résultats est basée sur les ANOVAs et leurs comparaisons post-hoc.

\subsection{Résultats}

Les moyennes et les écarts-types relatifs aux perceptions de MP, selon les conditions de séquence de momentum et d'écart de score, ainsi que les graphes des perceptions de MP pour chaque séquence de momentum sont reportés dans la Figure 3. Parce que le test de sphéricité était significatif $(p=0,003)$, la correction de Greenhouse-Geisser a été appliquée. Des effets principaux significatifs de la Séquence de momentum $\left(F_{(1,49)}=332,59, p<\right.$ 


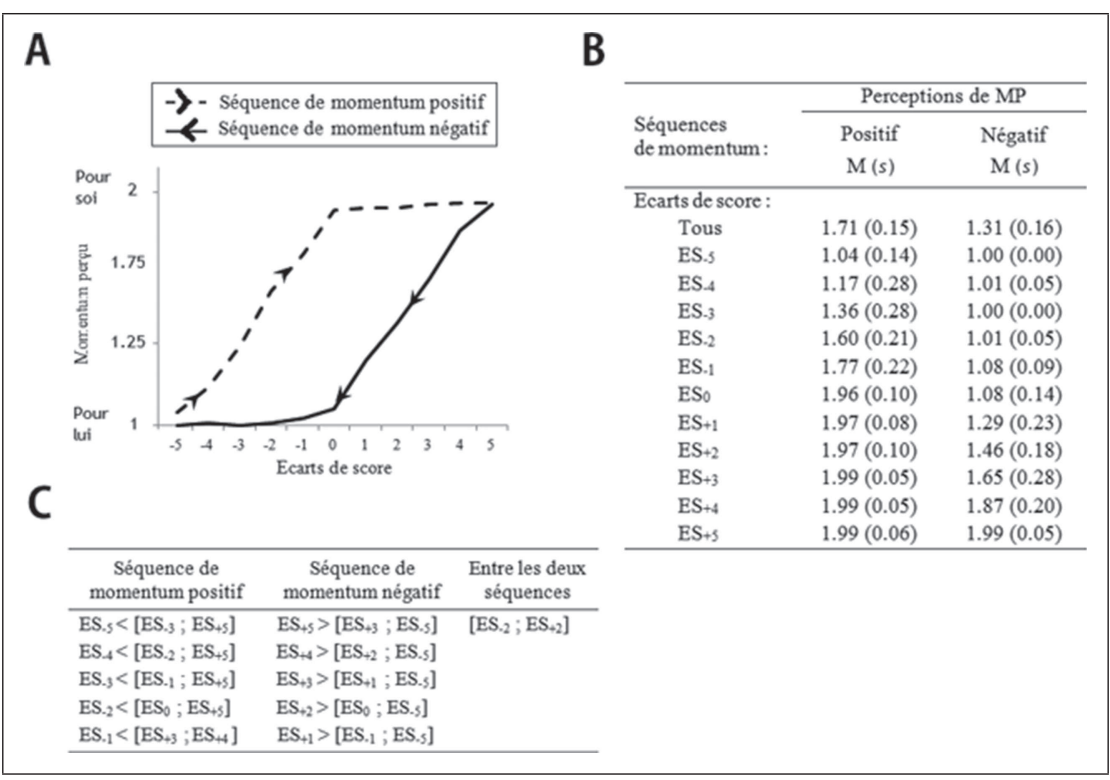

Figure 3. (A) Fluctuations, (B) moyennes, écart-types, et (C) différences entre les moyennes de perceptions de MP selon les séquences de momentum et les écarts de score. Figure 3. (A) Fluctuations, (B) means, standard deviations, and (C) differences in means of PM perceptions according to momentum sequences and score gaps.

$0,001, \eta^{2}$ partiel $\left.=0,87, \eta^{2}=0,87\right)$ et de l'Écart de score $\left(F_{(3.68,156.26)}=\right.$ 285,74, $p<0,001, \eta^{2}$ partiel $=0,85, \eta^{2}=0,73$ ) ainsi qu'un effet significatif d'interaction Séquence de momentum $\times$ Écart de score $\left(F_{(3.68,156.26)}=\right.$ $57,58, p<0,001, \eta^{2}$ partiel $\left.=0,54, \eta^{2}=0,15\right)$ ont été observés.

Dans la séquence de momentum positif, les perceptions de MP étaient significativement plus basses à $\mathrm{ES}_{-5}$ et à $\mathrm{ES}_{-4}$ qu'aux écarts de score supérieurs à $\mathrm{ES}_{-4}$ et à $\mathrm{ES}_{-3}$, respectivement ( $\left.p s<0,001\right)$; à $\mathrm{ES}_{-3}$ qu'à $\mathrm{ES}_{-1}$ $(p=0,008)$ et qu'aux écarts de score supérieurs à $\mathrm{ES}_{-1}(p s<0,001)$; à ES -2 qu'aux écarts de score supérieurs à ES-1 $(p s<0,001)$; à ES -1 qu'aux écarts de score $\mathrm{ES}_{+3}(p=0,03)$ et $\mathrm{ES}_{+4}(p=0,03)$ (voir Figure 3). Les écarts de score allant de $\mathrm{ES}_{0}$ à $\mathrm{ES}_{+5}$ n'étaient pas significativement différents ( $p s=$ 1,00) (voir Figure 3).

Dans la séquence de momentum négatif, les perceptions de MP étaient significativement plus élevées à $\mathrm{ES}_{+5}, \mathrm{ES}_{+4}, \mathrm{ES}_{+3}$, et $\mathrm{ES}_{+2}$ qu'aux écarts de score inférieurs à $\mathrm{ES}_{+4}, \mathrm{ES}_{+3}, \mathrm{ES}_{+2}$, et à $\mathrm{ES}_{+1}$, respectivement $(p s<$ $0,001)$; à $\mathrm{ES}_{+1}$ qu'aux écarts de score $\mathrm{ES}_{-1}(p=0,01), \mathrm{ES}_{-2}(p=0,002)$, 
$\mathrm{ES}_{-3}(p<0,001), \mathrm{ES}_{-4}(p=0,002)$, et $\mathrm{ES}_{-5}(p<0,001)$ (voir Figure 3$)$. Les écarts de score allant de $\mathrm{ES}_{0}$ à $\mathrm{ES}_{-5}$ n'étaient pas significativement différents (ps $>0,98)$ (voir Figure 3).

Finalement, la comparaison des deux séquences de momentum à des écarts de score identiques a révélé que les perceptions de MP étaient significativement plus élevées en séquence de momentum positif qu'en séquence de momentum négatif seulement aux écarts de score $\mathrm{ES}_{-2}(p<$ $0,001), \mathrm{ES}_{-1}(p<0,001), \mathrm{ES}_{0}(p<0,001), \mathrm{ES}_{+1}(p<0,001)$ et $\mathrm{ES}_{+2}(p=$ 0,007) (voir Figure 3).

\subsection{Discussion}

Un premier objet de la présente étude était de tester les propriétés de non-linéarité et de dépendance à l'histoire du MP. Les résultats ont montré l'existence (a) d'un effet d'interaction Séquence de momentum $\times$ Écart de score significatif en montrant des différences significatives entre les séquences de momentum vers le milieu de ces séquences seulement (c'est-à-dire, entre $\mathrm{ES}_{-2}$ et $\mathrm{ES}_{+2}$ ), (b) d'un effet principal significatif de la Séquence de momentum en montrant que les perceptions de MP étaient plus élevées en momentum positif qu'en momentum négatif, et (c) d'un effet principal significatif de l'Écart de score en montrant des variations abruptes dans les perceptions de MP en début de chaque séquence de momentum. Par conséquent, les perceptions de MP ont montré un patron d'hystérésis négative (voir Tableau 2). Comme l'ont supposé Gernigon et al. (2010), ce patron dynamique pourrait refléter la propension des individus expérimentant le MP à se projeter dans un scénario futur (par ex., la victoire, la défaite, etc.).

Un second objet d'étude avait pour but d'examiner si la dynamique des perceptions de MP montrait une asymétrie entre le MP positif et le MP négatif. Parce que l'effet d'interaction Séquence de momentum $\times$ Écart de score a montré que les différences significatives entre les deux séquences de momentum ont concerné un nombre égal de paliers d'écarts de score de part et d'autre de l'écart de score $\mathrm{ES}_{0}$ (correspondant à un état d'égalité au score entre les deux joueurs), aucune asymétrie entre les deux formes de MP n'a pu être identifiée.

La présente étude présente quelques limites méthodologiques. Premièrement, les étudiants qui ont été volontaires pour participer à cette étude n'étaient pas des pratiquants du tennis de table, il est donc tout à fait possible d'envisager qu'ils aient rencontré des difficultés à se projeter dans le scénario sportif visionné. Deuxièmement, seuls quatre 
items de perceptions de MP seulement ont été utilisés au cours de cette étude, ce qui peut sembler réducteur au regard du questionnaire des perceptions de MP (voir Vallerand et al., 1988). Cependant, cette contrainte méthodologique visait d'abord à limiter l'effet potentiel d'une interruption de la tâche expérimentale (c'est-à-dire, de la séquence de momentum) sur les perceptions de MP des participants (voir Briki, Doron et al., 2014).

\section{3. ÉTUDE 2}

Considérant les résultats de l'étude précédente et ses limites, les objets de cette seconde étude étaient de vérifier que les perceptions de MP révélaient des propriétés de non-linéarité et de dépendance à l'histoire au travers de l'émergence d'un patron d'hystérésis négative et d'examiner l'effet d'asymétrie potentielle entre le MP positif et le MP négatif. Afin de répondre aux critiques qui ont été évoquées dans l'étude précédente, quelques mesures ont été adoptées. Premièrement, le degré d'immersion des participants dans les situations virtuelles projetées a été contrôlé. Deuxièmement, d'autres items de perceptions de MP issus du questionnaire de Vallerand et al. (1988) ont été utilisés au cours de la présente étude.

Les hypothèses étaient basées sur les résultats de l'étude précédente. Étant donné que les perceptions de MP ont révélé un patron d'hystérésis négative, il était attendu que les perceptions de MP étudiées dans la présente étude montrent un même patron dynamique. Puis, parce que l'Étude 1 n'a pas conforté l'hypothèse de l'asymétrie entre le MP positif et le MP négatif (Gernigon et al., 2010), aucune attente n'a pu être formulée à ce propos.

\subsection{Méthode}

\subsubsection{Participants et procédure}

Soixante-huit étudiants (femmes $=36$; hommes $=32$ ) de la faculté des sciences du sport de l'Université Montpellier I ont été volontaires pour participer à cette étude en l'échange d'aucune rémunération. Le recrutement de ces participants s'était réalisé sur la base de leur disponibilité. Tous les participants pratiquaient le sport en compétition dans des activités différentes de celles du tennis de table. Ils étaient âgés en moyenne de 23.6 ans $(s=6.96)$. La procédure menée au cours de cette étude était strictement identique à celle employée au cours de l'étude précédente. 
Parmi les 68 participants de la présente étude, seuls 29 d'entre eux (femmes $=$ 15 ; hommes $=14$ ), âgés en moyenne de 22,5 ans $(s=5,31)$, ont été sélectionnés pour l'exécution des analyses parce que ces participants avaient fourni une réponse strictement supérieure à 4 à l'item de contrôle : "Dans quelle mesure, je suis parvenu à m'imaginer, moi-même, en train de vivre cette manche décisive ? " . Cette question était mesurée sur une échelle de type Lickert en sept points, allant de « pas du tout » (1) à «très fortement » (7). Cet item de contrôle avait pour but de contrôler le degré d'immersion des participants dans les situations compétitives proposées ; cet item était inspiré de ceux utilisés dans de récents travaux de psychologie sociale portant sur le MP (voir Briki, Den Hartigh, Markman, \& Gernigon, 2014 ; Briki, Doron et al., 2014). Parmi les participants retenus, 15 d'entre eux (femmes $=8$; hommes $=7$ ) ont intégré la condition de momentum positif alors que 14 d'entre eux (femmes $=7$; hommes $=7$ ) ont fait partie de la condition de momentum négatif.

\subsubsection{Mesures et analyses}

Les modalités de mesures et d'analyses utilisées au cours de la présente étude ont été identiques à celles qui ont été employées dans l'Étude 1. Les perceptions de MP étaient mesurées par quatre items présentés de manière randomisée. Ces items étaient les suivants : "Qui te semble être le plus confiant? »; «Qui te semble être le plus motivé ? »; «Qui te semble être le plus découragé ? » (item au codage inversé); «Qui te semble être le plus inquiet ${ }^{3}$ ? » (item au codage inversé) ( $\alpha=0,98$; IC $95 \%$ $[0,97 ; 0,99])$. Ces items ont été aussi choisis en raison de leur compatibilité avec le contexte du tennis de table.

\subsection{Résultats}

Les moyennes et les écarts-types relatifs aux perceptions de MP, selon les conditions de séquence de momentum et d'écart de score, ainsi que les graphes des perceptions de MP pour chaque séquence de momentum sont reportés dans la Figure 4. Étant donné que le test de sphéricité était significatif $(p<0,001)$, la correction de Greenhouse-Geisser a été appliquée. Des effets principaux significatifs de la Séquence de momentum $\left(F_{(1,27)}=\right.$ 640,06, $p<0,001, \eta^{2}$ partiel $\left.=0,96, \eta^{2}=0,51\right)$ et de l'Écart de score $\left(F_{(3,20,86,28)}=73,21, p<0,001, \eta^{2}\right.$ partiel $\left.=0,73, \eta^{2}=0,96\right)$ ainsi qu'un effet significatif d'interaction Séquence de momentum $\times$ Écart de score $\left(F_{(3,20,86,28)}=42,55, p<0,001, \eta^{2}\right.$ partiel $\left.=0,61, \eta^{2}=0,30\right)$ ont été trouvés.

${ }^{3}$ L'item d'《 anxiété perçue 》, ne figurant pas dans le questionnaire de Vallerand et al. (1988), a été proposé et utilisé par Miller et Weinberg (1991) parce que cette variable pourrait capturer le MP. Des analyses de corrélation complémentaires ont révélé que l'item d'anxiété perçue (inversement codé) était très fortement corrélé aux items de perceptions de confiance $(r=0,95, p<0,001)$, de motivation $(r=0,93, p<0,001)$ et de découragement (inversement codé) $(r=0,94, p<0,001)$. 


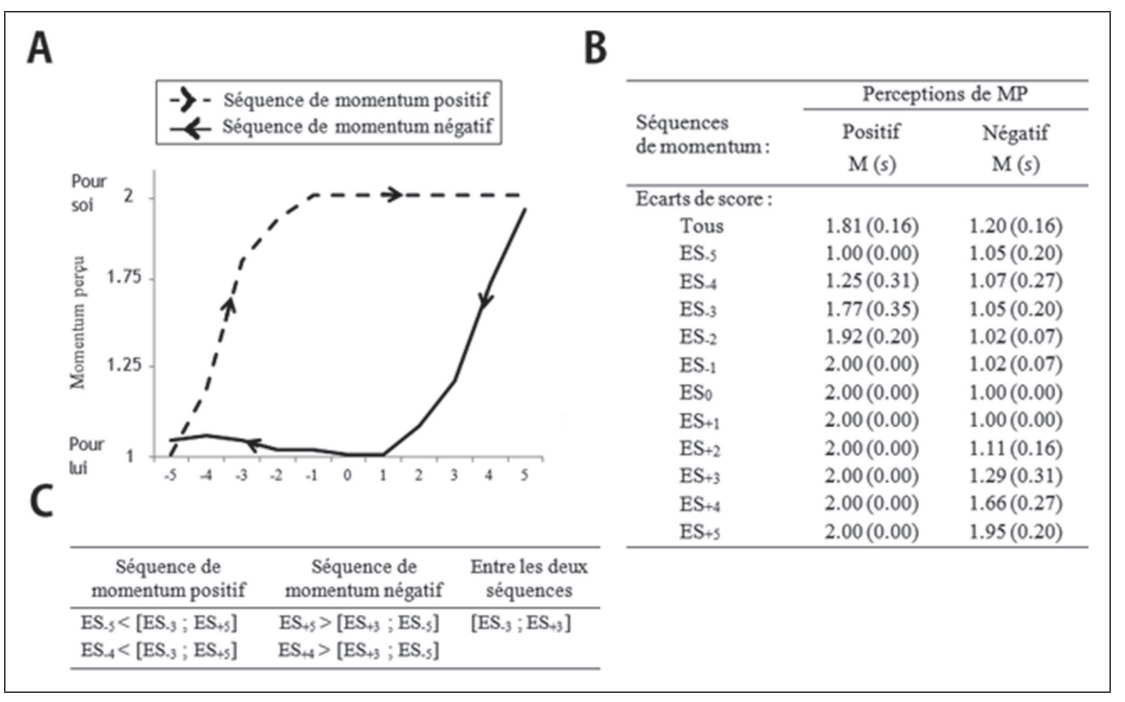

Figure 4. (A) Fluctuations, (B) moyennes, écart-types, et (C) différences entre les moyennes de perceptions de MP selon les séquences de momentum et les écarts de score. Figure 4. (A) Fluctuations, (B) means, standard deviations, and (C) differences in means of PM perceptions according to momentum sequences and score gaps.

Dans la séquence de momentum positif, les perceptions de MP étaient significativement plus basses à $\mathrm{ES}_{-5}$ et à $\mathrm{ES}_{-4}$ qu'aux écarts de score supérieurs à $\mathrm{ES}_{-4}(p s<0,001)$ (voir Figure 4). Les écarts de score allant de $\mathrm{ES}_{-3}$ à $\mathrm{ES}_{+5}$ n'étaient pas significativement différents $(p s>0,83)$ (voir Figure 4).

Dans la séquence de momentum négatif, les perceptions de MP étaient significativement plus élevées à $\mathrm{ES}_{+5}$ qu'aux écarts de score inférieurs à $\mathrm{ES}_{+4}(p s<0,001)$; à ES $+4 q$ qu' $\mathrm{ES}_{+3}(p=0,03)$ et qu'aux écarts de score inférieurs à $\mathrm{ES}_{+3}(p s<0,001)$ (voir Figure 4). Les écarts de score allant de $\mathrm{ES}_{+3}$ à $\mathrm{ES}_{-5}$ n'étaient pas significativement différents $(p s>0,49)$ (voir Figure 4).

Finalement, la comparaison des deux scénarios à des écarts de score identiques a révélé que les perceptions de MP étaient significativement plus élevées en séquence de momentum positif qu'en séquence de momentum négatif, pour les écarts de score $\mathrm{ES}_{-3}, \mathrm{ES}_{-2}, \mathrm{ES}_{-1}, \mathrm{ES}_{0}, \mathrm{ES}_{+1}, \mathrm{ES}_{+2}$ et $\mathrm{ES}_{+3}$ $(p s<0,001)$ (voir Figure 4). 


\subsection{Discussion}

Le premier objet de cette seconde étude était de vérifier si les perceptions de MP montraient un patron d'hystérésis négative, lequel combine les propriétés de non-linéarité et de dépendance à l'histoire. Comme dans l'Étude 1, les résultats de la présente étude ont rapporté l'ensemble des critères requis à l'identification du patron d'hystérésis négative (voir Tableau 2), renforçant la spéculation de Gernigon et al. (2010) envisageant le MP comme un phénomène spécifiquement tourné vers un futur anticipé. Le second objet de cette étude a consisté à explorer l'effet potentiel d'asymétrie entre le MP positif et le MP négatif. Comme dans l'Étude 1, parce que les différences significatives entre les deux séquences de momentum ont concerné un nombre identique de paliers d'écarts de score autour de l'écart de score $\mathrm{ES}_{0}$, les dynamiques des MP positif et négatif peuvent être considérées comme symétriques.

\section{DISCUSSION GÉNÉRALE}

La présente série d'études avait pour but d'examiner si les perceptions de MP montraient des signatures typiques des systèmes dynamiques telles la non-linéarité et la dépendance à l'histoire. Cette série d'études avait aussi pour but de comparer la dynamique des MP positif et négatif. Compte tenu du travail de Gernigon et al. (2010) et de Briki, Den Hartigh, Markman et al. (2013), il était attendu que la dynamique des perceptions de MP révèle : (a) des phénomènes d'anticipation et/ou de persistance durant les séquences de momentum, et (b) des effets asymétriques entre les deux formes de MP dans le sens où le MP négatif se développerait plus tôt que le MP positif. De telles hypothèses ont été testées en respectant les contraintes méthodologiques du paradigme de Haken et al. (1985).

Les deux études ont supporté l'hypothèse selon laquelle la dynamique du MP est non-linéaire et dépendante de l'histoire des événements, en révélant un patron d'hystérésis négative qui reflète un phénomène d'anticipation dans les deux séquences de momentum (Gernigon et al., 2010). D'un point de vue psychologique, ce patron dynamique indiquerait que les acteurs placés en situations d'accomplissement virtuelles auraient tendance à se projeter dans l'avenir probable de la situation sur la base du moindre indice contradictoire avec l'état actuel de la situation (Gernigon et al., 2010). 
La cohérence des résultats de notre travail avec ceux de Gernigon et al. (2010) supporte l'idée que les perceptions de MP peuvent être des paramètres d'ordre fiables du MP (Briki, Den Hartigh, Markman et al., 2013). Par ailleurs, l'observation d'un patron d'hystérésis négative et non d'un patron de limite critique - tel qu'il a été observé dans l'étude de Briki, Den Hartigh, Markman et al. (2013) - renforce l'hypothèse de Briki, Den Hartigh, Markman et al. (2013) selon laquelle les incohérences de résultats observées entre leur travail et celui de Gernigon et al. (2010) pourraient être imputées à l'existence d'un effet différencié du type d'implication dans la tâche (c'est-à-dire, acteur $v s$ observateur). Dans cette perspective, Briki, Den Hartigh, Markman et al. (2013) ont supposé que les participants qui étaient placés en position d'acteur projeté dans des situations virtuelles pourraient avoir tendance à réaliser la tâche depuis le point de vue du pur observateur. En conséquence, ces auteurs ont spéculé que le patron d'hystérésis négative refléterait la dynamique des perceptions du MP depuis la position de l'observateur.

La comparaison des dynamiques des MP n'a pas mis en évidence des effets d'asymétrie entre les deux formes de MP. D'un point de vue dynamique, ceci suggère que le MP positif et le MP négatif représentent tous deux des attracteurs de même force. Cependant, notre présent travail comporte une limite majeure ayant pu influencer les résultats observés : les deux études étaient basées sur des situations virtuelles et il est donc possible que ces situations aient pu (a) encourager une implication dans la tâche en tant qu'observateur (Briki, Den Hartigh, Markman et al., 2013) et (b) réduire l'impact des séquences de momentum sur la sphère perceptive et affective des participants (Gernigon et al., 2010). Des travaux ultérieurs devraient donc placer les participants en situations d'observateur d'une compétition impliquant des athlètes en situations réelles de production de performance (voir Briki, Den Hartigh, Markman, \& Gernigon, 2014). Ceci aurait pour but de tester les hypothèses selon lesquelles le patron d'hystérésis négative reflète la dynamique du MP depuis le point de vue des observateurs (Briki, Den Hartigh, Markman et al., 2013) et le MP est un phénomène asymétrique (Adler, 1981 ; Briki, Doron et al., 2014 ; Briki, Den Hartigh, Markman et al., 2013 ; Gernigon et al., 2010). Des études ultérieures devraient aussi conforter l'hypothèse selon laquelle le patron de limite critique dans la dynamique des perceptions de MP est spécifique à une implication dans la tâche en tant qu'acteur en situations réelles de production de performance (Briki, Den Hartigh, Markman et al., 2013).

Reçu 31 mars 2013.

Révision acceptée le 24 juin 2014. 


\section{BIBLIOGRAPHIE}

Adler, P. (1981). Momentum: A theory of social action. Beverly Hills, CA: Sage.

Baumeister, R. F., Bratslavsky, E., Finkenauer, C., \& Vohs, K. D. (2001). Bad is stronger than good. Review of General Psychology, 5, 323-370. doi :10.1037/10892680.5.4.323.

Briki, W., Den Hartigh, R. J. R., Bakker, F. C., \& Gernigon, C. (2012). The dynamics of psychological momentum: A quantitative study in natural sport situations. International Journal of Performance Analysis in Sport, 12, 573-592.

Briki, W., Den Hartigh, R. J. R., Hauw, D., \& Gernigon, C. (2012). A qualitative exploration of the psychological contents and dynamics of momentum in sport. International Journal of Sport Psychology. 43, 365-384. doi: 10.7352/IJSP 2012.43.365. Briki, W., Den Hartigh, R. J. R., Markman, K. D., \& Gernigon, C. (2014). How do supporters perceive positive and negative psychological momentum changes during a simulated cycling competition? Psychology of Sport and Exercise, 15, 216-221. http:// dx.doi.org/10.1016/j.psychsport.2013.11. 006.

Briki, W., Den Hartigh, R. J. R., Markman, K. D., Micallef, J.-P., \& Gernigon, C. (2013). How psychological momentum changes in athletes during a sport competition. Psychology of Sport and Exercise, 14, 389-396. http://dx.doi.org/ 10.1016/j.psychsport.2012.11.009.

Briki, W., Doron, J., Markman, K. D., Den Hartigh, R. J. R., \& Gernigon, C. (2014). Differential reactions of actors and observers to the triggering and interruption of psychological momentum. Motivation and Emotion, 38, 263-269. doi : 10.1007/s11031013-9372-3.
Brislin, R. W., Lonner, W. J., \& Thorndike, R. M. (1973). Cross-cultural research methods. New York : Wiley.

Gernigon, C., Briki, W., \& Eykens, K. (2010). The dynamics of psychological momentum in sport: The role of ongoing history of performance patterns. Journal of Sport \& Exercise Psychology, 32, 377-400.

Haken, H., Kelso, J. A. S., \& Bunz, H. (1985). A theoretical model of phase transitions in human hand movements. Biological Cybernetics, 51, 347-356.

Jones, M. I., \& Harwood, C. (2008). Psychological momentum within competitive soccer: Players' perspectives. Journal of Applied Sport Psychology, 20, 57-72. doi:10.1080/10413200701784841.

Kahneman, D., \& Tversky, A. (1979). Prospect theory: An analysis of decision under risk. Econometrica, 47, 263-291.

Kelso, J. A. S. (1995). Dynamic patterns: The self-organization of brain and behavior. Cambridge, MA: MIT Press.

Markman, K. D., \& Guenther, C. L. (2007). Psychological momentum: Intuitive physics and naive beliefs. Personality and Social Psychology Bulletin, 33, 800-812. doi: 10.1177/0146167207301026.

Miller, S., \& Weinberg, R.S. (1991). Perceptions of psychological momentum and their relationship to performance. The Sport Psychologist, 5, 211-222.

Nowak, A., \& Vallacher, R. R. (1998). Dynamical social psychology. New York: Guilford Press.

Nowak, A., Vallacher, R. R., \& Zochowski, M. (2005). The emergence of personality: Dynamic foundations of individual variation. Developmental Review, 25, 351-385. 
doi: 10.1016/j.dr.2005.10.004. ISSN : 02732297.

Perreault, S., Vallerand, R. J., Montgomery, D., \& Provencher, P. (1998). Coming from behind : On the effect of psychological momentum on sport performance. Journal of Sport \& Exercise Psychology, 20, 421-436.

Tabachnick, B. G., \& Fidell, L. S. (2007). Using multivariate statistics (5th ed.). Boston: Pearson Education.
Tuller, B., Case, P., Ding, M., \& Kelso, J. A. S. (1994). The nonlinear dynamics of speech categorization. Journal of Experimental Psychology. Human Perception and Performance, 20, 3-16.

Vallerand, R. J., Colavecchio, P. G., \& Pelletier, L. G. (1988). Psychological momentum and performance inferences: A preliminary test of the antecedents-consequences psychological momentum model. Journal of Sport \& Exercise Psychology, 10, 92-108. 